CSR-P-69-24 September 1969

\title{
MATHEMATICAL MODELS OF MAGNETOSPHERIC CONVECTION AND ITS COUPLING \\ TO THE IONOSPHERE
}

\author{
Vytenis M. Vasyliunas \\ Department of Physics and Center for Space Research \\ Massachusetts Institute of Technology \\ Cambridge, Massachusetts
}

Paper presented at the Summer Advanced Study Institute - Earth's Particles and Fields 1969, Santa Barbara, California, August 1969. To be published in Particles and Fields in the Magnetosphere, edited by B.M. McCormac (D. Reidel Publishing Company, Dordrecht Holland), 1970. 
INTRODUCTION

The phase "magnetospheric model" can have several different meanings. Here I am using the term "model" in the sense described by PARKER (1968): "We construct idealized and simplified theoretical models for the purpose of demonstrating how the basic laws of physics lead to a certain observed effect." A model in this sense is a solution of the equations that describe the system under consideration. Obtaining an exact solution of the equations governing a system as complex as the magnetosphere is clearly impossible, and to construct a theoretical model the equations must be (often drastically) simplified to the point of tractability. The aim is to isolate those aspects of the physical situation that are essential to the particular phenomenon one is attempting to understand. One thus proceeds by solving the basic equations under a variety of simplifying assumptions and noting what assumptions are required to reproduce the essential features of the phenomenon under study. Of course, no model will predict in precise quantitative detail all the features of the observations, but then our primary goal is understanding, not forecasting.

In this paper I discuss theoretical models (in the sense described above) of some aspects of magnetospheric convection and its effects on charged particle populations. Starting with the classic work of AXFORD and HINES (1961), the concepts and consequences of magnetospheric convection have been widely discussed in qualitative 
form (see, e.g., reviews by AXFORD, 1969 and KENNEL, 1969); the task now is to express some of these ideas in quantitative form as appropriate solutions of the basic equations. First I will formulate the mathematical problem of calculating a model of steady state convection and the associated particle distribution. Then I will discuss solutions of several parts of the problem (a solution of the complete problem has not yet been obtained) and their relation to observed phenomena.

\section{MAGNETOSPHERIC CONVECTION AS A MATHEMATICAL PROBLEM}

in principle, magnetospheric convection could be treated with magnetohydrodynamic equations, relating the flow velocity to the stresses present in the plasma; this approach has been highly sucessful in treating the solar wind. The flow speeds associated with convection, however, are expected to be very small compared to propagation speeds of waves in the magnetospheric medium, and the characteristic flow times should thus be very long compared to the travel time of a signal between the ionosphere and the equatorial plane (except possibly in the region of "open" field lines deep within the magnetotail). Thus we expect the plasma at any point of a magnetic flux tube within the magnetosphere to remain continually "adjusted" to the boundary conditions at the feet of that flux tube on the ionosphere. With the expected close coupling between the magnetosphere and the ionosphere, two media with very different dynamical 
properties, it proves convenient to formulate the convection problem not in terms of the dynamical concepts of flow and stress but in terms of electric field and current (fundamentally, of course, these two modes of treating the problem are equivalent, since in a plasma there is a close connection between the flow and the electric field and between the stress and the electric current).

The electric field and the charged particle distribution in the magnetosphere can both be simultaneously calculated from a closed self-consistent chain of equations, first introduced by FEJER (1964) and later used by SWIFT (1967, 1968). The complete calculation is outlined in Figure 1. Since it is a closed loop, we can break into it at any point; it is convenient to start with the electric field. Let us provisionally suppose, then, that we know the electric field configuration in the magnetosphere:

(a) First link: with knowledge of the electric field, we calculate the motion and distribution of protons and electrons in the magnetosphere, and hence in particular the total plasma pressure at any point;

(b) Second link: from the plasma pressure gradients we calculate the components of the electric current perpendicular to the magnetic field.

(c) Third link: by calculating the divergence of the perpendicular current and averaging over each flux tube, we obtain the field-aligned currents flowing between the magnetosphere and the ionosphere. 
(d) Fourth link: from the requirement that these field aligned currents be closed by perpendicular ohmic currents in the ionosphere, we obtain the configuration of the electric field in the ionosphere.

(e) Fifth and final link: the ionospheric electric field can be mapped into the magnetosphere, and the requirement that it agree with the magnetospheric electric field assumed at the outset determines the field and thus closed the system of equations.

Let us now examine in more detail the individual links of the chain.

(a) Calculation of the charged particle distribution in a given configuration of electric (and magnetic) fields is an extensively studied and familiar process. The simplest method, if only adiabatic processes are considered, is to calculate single-particle trajectories (see, e.g., ALFVÉN, 1939; TAYLOR and HONES, 1965; KAVANAGH et al., 1968) and apply Liouville's theorem; or one may, by suitable transformations of the Boltzmann equation, develop a transport equation (e.g. SISCOE, 1964; VASYLIUNAS, 1969), with the capability of including non-adiabatic scattering, acceleration, and loss processes. As a boundary condition, it is necessary to specify the particle population at the boundary of the region under study, at those points of the boundary where particles can move into the region. Usually the region studied is the magnetosphere out to some 10-15 $\mathrm{R}_{\mathrm{e}}$ on the night side and the electric field is such that particles move from the 
magnetotail toward the earth; then it is necessary to specify the particle population at the near-earth end of the magnetotail, and the observed plasma sheet population is a reasonable choice.

(b) The calculation of the perpendicular current from the total pressure can be looked at in two equivalent ways. We may write down the momentum conservation equation for the plasma, which in the approximation of flow speed very small compared to particle thermal speed (valid throughout the magnetosphere beyond the plasmapause, except possible within the neutral sheet) reduces to

$$
\nabla \cdot \underset{\sim}{\stackrel{P}{\sim}}=\frac{1}{\mathrm{C}} \underset{\sim}{\mathrm{J}} \times \underset{\sim}{\mathrm{B}}
$$

where $\underset{\eta}{\mathrm{q}}$ is the total (proton plus electron) pressure tensor and the rest of the symbols have their usual meaning (Gaussian units). Solving for the perpendicular component of the current density yields, if the pressure is isotropic,

$$
\beth_{\perp}=\frac{C \stackrel{C}{\mathrm{~B}} \times \nabla P}{B^{2}}
$$

and if the pressure is anisotropic but field-aligned

$$
J_{\perp}=\frac{\underset{\sim}{\mathrm{B}} \times\left[\nabla P_{\perp}+\left(P_{11}-P_{\perp}\right) \nabla B / B\right]}{B^{2}+4\left(P_{\perp}-P_{11}\right)}
$$

Equivalently, we may sum the currents due to all the single particle 
gradient, curvature, and magnetization drifts, obtaining the same result (see, e.g., PARKER, 1957). Note that $J_{\mathcal{L}}$ depends only on the total pressure and the magnetic field and has no explicit dependence on the electric field or the detailed energy spectra of the particles; given only the configuration of the magnetic field and the total pressure, however obtained, the perpendicular current is uniquely determined. (It is assumed here that the general configuration of the magnetic field is known from observation or field models, and that the detailed magnetic effects of $\underset{\beth}{J}$ can be neglected.)

(c) The current density given by equation (2) or (3) in general has a non-zero divergence. The total current density, however, must have zero divergence, even if the convection is assumed to be highly non-steady; it is readily shown that under magnetospheric conditions the charge accumulation implied by the divergence of $J_{\perp}$ alone would lead to electric field changes on a time scale of milliseconds (a not surprising result if one recalls that allowing $\nabla \cdot \underset{\sim}{J} \neq 0$ is the same as keeping the displacement current term in Maxwell's equations). Hence in all magnetospheric processes other than high-frequency plasma waves $\nabla \cdot \underset{\tau}{J}=0$ and the divergence of $J_{\perp}$ must be cancelled by the divergence of a magnetic field aligned current density $J_{\text {. }}$ From the divergence of equation (2) or (3) we thus obtain the derivative of $J_{\text {" }}$ along the magnetic field direction:

$$
\frac{\partial}{\partial \ell}\left(\frac{J_{H}}{B}\right)=\frac{2 \mathrm{C}\left(\mathbb{Z}_{\mathrm{B}} \times \nabla \mathrm{B}\right) \cdot \nabla \mathrm{P}}{\mathrm{B}^{4}}
$$

for the isotropic pressure, or 


$$
\frac{\partial}{\partial \ell}\left(\frac{J_{11}}{B} \xi\right)=\frac{C(\stackrel{B}{\imath} \times \nabla B) \cdot \nabla\left(P_{\perp}+P_{11}\right)}{\xi B^{4}}
$$

where $\xi \equiv 1+4 \pi\left(P_{\perp}-P_{11}\right) / B^{2}$ for the general case. As expected from a simple picture of particle drifts, field-aligned currents are present whenever the pressure gradient has a component along the direction of the $\nabla B$ drift. If, for example, we have a region of enhanced pressure within a narrow longitude sector, a field-aligned current flows into the ionosphere at its western edge and out of the ionosphere at its eastern edge. The total current density flowing in or out of the ionosphere at any point must be found by integrating equation (4) or (5) along the field line; with the isotropic pressure, which must be constant along the field line, equation (4) can be integrated explicitly (VASYLIUNAS, 1969), yielding

$$
J_{n}=\frac{B_{m}}{2 B_{e}} c\left(\nabla P \times \frac{\mathrm{B}_{e}}{B_{e}}\right) \cdot \nabla f \frac{\mathrm{dl}}{B}
$$

where $\underset{\sim}{\mathrm{B}}$ is the magnetic field at the equator, $\mathrm{B}_{\mathrm{m}}$ the field magnitude at the ionosphere ("mirror field"), the gradients are with respect to the equatorial coordinates of the field line, the integration is along the entire length of the field line above the ionosphere, and $J_{n}$ is per unit area of the ionosphere and positive if into the ionosphere.

(d) Continuity of current in the ionosphere requires that 


$$
\nabla \cdot \frac{I}{2}=J_{n} \sin x
$$

where $I_{\sim}$ is the height-integrated horizontal current density, $x$ is the inclination of the magnetic field, and $J_{\text {" }}$ is the field-aligned current density flowing into the ionosphere. If the electric field $\underset{\sim}{\mathrm{F}}$ at the top of the ionosphere has no horizontal variation except on a sufficiently long scale $(\gtrsim 10 \mathrm{~km})$, $\underset{\sim}{\mathrm{I}}$ can be related to $\underset{\sim}{\mathrm{E}}$ by the equation

$$
\underset{\sim}{I}=\sum_{\approx} \cdot(E+\stackrel{V}{\mathrm{~V}} \cdot \underset{\sim}{\mathrm{C}})
$$

where $\mathrm{V}_{\mathrm{n}}$ is the velocity of the neutral gas in the ionosphere (commonly assumed to corotate with the earth) and $\sum_{\approx}$ is the familiar height-integrated conductivity tensor (see, e.g., FEJER, 1953, 1964). Inserting (8) into (7) provides a differential equation from which $\underset{\sim}{E}$ may be obtained if $J_{n}$ is known. $\quad\left(\frac{\partial R}{\partial t}=-C \nabla \times \underset{\sim}{E}\right.$ must be known or set to zero.) Note that, given the conductivity and the neutral gas motion, $\underset{\sim}{\mathrm{E}}$ is uniquely determined by $J_{"}$ (or vice versa) and does not explicitly depend on any other magnetospheric variables such as pressure gradients.

(e) The electric field at the top of the ionosphere and the electric field within the magnetosphere are related by the generalized Ohm's law, and thus either can be calculated from the other. For quasi-steady convection, in which the electric field can be derived from a potential, one needs to calculate the potential drop along the field line from the ionosphere to the equatorial plane. A common 
assumption that appears to be at least a good first approximation is that the conductivity along the field lines is high enough so that this potential drop can be neglected and the potential simply mapped out from the ionosphere into the magnetosphere. Anomalous resistivity caused by instabilities (see, e.g., KENNEL, 1970) may produce significant potential drops which can, however, in principle be calculated if $\mathrm{J}_{n}$ is known.

This entire calculation should provide a reasonable complete model of the processes associated with convection within the "closed" part of the magnetosphere, in which the magnetic field lines connect from one hemisphere to the other. The "open" field lines that extend far out into the magnetotail must ultimately enter either the interplanetary medium, as in the models of DUNGEY (1961) and his followers, or/and a magnetosphere boundary layer within which a viscous drag (AXFORD and HINES, 1961; AXFORD, 1964) or some other tangential stress responsible for maintaining the magnetotail (AXFORD et al., 1965; SISCOE, 1966) occurs. On these magnetic field lines, the electric field is determined by processes associated with the field line merging or the tangential stress, whose physics is at present largely unknown; it is this electric field, however, that sets the entire convection system going and thus it may appropriately be called the driving field. Equations (7) and (8) are still applicable and, given the driving field, serve to determine the field-aligned current den-

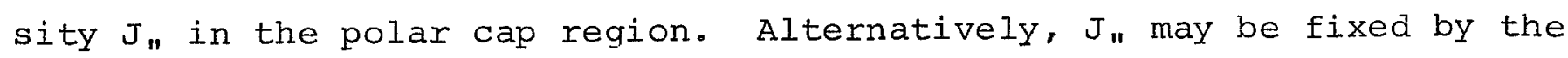


field line merging or tangential stress processes, in which case it is the driving current, setting up the convection; equations (7) and (8) then determine the associated electric field. Finally, if the calculation is to be restricted to the closed field line region only, the driving field must still be partially specified as the boundary condition on $\underset{\sim}{E}$ now required to solve equations (7) and (8).

In summary, understanding the origin of the driving electric field (or current) and the origin of the plasma sheet is a prerequisite for a complete model of magnetospheric convection, and such understanding we do not now possess. However, the properties of the plasma sheet particles are well known from observation and, as will appear shortly, crude but useful models of the driving field can be guessed; given both of these, the set of equations described here allows one to construct a model of the convection within the closed part of the magnetosphere, including the effects of interactions between the plasma, the electric fields, and the ionosphere; this now requires nothing more than solution of a well-defined computational problem! Even with drastic simplifications, the problem is extremely complicated and I have not yet obtained a solution of the complete selfconsistent chain. Individual links of the chain, however, have been studied and useful results obtained. Models of the first link, particle distribution in given electric fields, have been constructed by TAYLOR and HONES (1965) and KAVANAGH et al. (1968) from a strictly adiabatic viewpoint. I have studied the same problem with the 
inclusion of non-adiabatic effects; the calculation is published elsewhere (VASYLIUNAS, 1969), and the basic physical ideas and some results have been described by KENNEL (1969). In the following section I consider simple models of another link: the connection between field-aligned currents and electric fields in the ionosphere.

\section{A MODEL ELECTRIC FIELD AND CURRENT}

\section{SYSTEM IN THE IONOSPHERE}

The basic assumption of the convection models first introduced by AXFORD and HINES (196I) and DUNGEY (196I) is that magnetic field lines from the polar caps (assumed to extend into interplanetary space or a viscous drag boundary layer) move, at least partially, with the solar wind. This implies an electric field over the polar cap (the driving field) directed in a general way from dawn to dusk; thus on the polar cap boundary the electric potential (assuming now that any variations in the convection pattern are slow enough so that induction fields can be neglected) is positive on the dawn side and negative on the dusk side. To obtain a very simple model, we approximate the polar cap boundary be a circle of constant latitude $\lambda=72^{\circ}$ and assume the potential on the boundary

$$
\Phi(\lambda, \phi)=\Phi_{0} \sin \phi \text { at } \lambda=72^{\circ}
$$

where $\phi$ is the longitude measured eastward from the midnight meridian 
and $\Phi_{0}$ is a constant. Since we are now interested in ionospheric effects on convection, we neglect particle pressure gradient effects and solve equations (7) - (8) with $J_{n}=0$ to find the potential at lower latitudes, with (9) as the boundary condition. The solutions are obtained in a frame of reference rotating with the earth and ${ }_{V_{n}}$ in $(8)$ is set to zero. For simplicity, symmetry between the two hemispheres is assumed and day-night variations in the conductivity are neglected (some of the effects neglected here are included in a similar calculation recently done independently by WOLF, 1969). The conductivity model of FEJER (1953, 1964) is used, with a value of 3.5 for the ratio of height-integrated Hall to Pedersen conductivities.

The resulting potential configuration in the ionosphere is shown in Figure 2; it looks rather similar to the configuration inferred from DP2 magnetic variations (OBAYASHI and NISHIDA, 1968). To obtain a solution within the polar cap, it was assumed that $J_{\|}=0$ also over the polar cap, for lack of any better choice. The field-aligned currents (which necessarily must be present; cf. VASYLIUNAS, 1968) are then confined to the polar cap boundary; for the case shown in Figure 2, the current density is

$$
J_{n}(\lambda, \phi)=\alpha\left(\Phi_{0} \Sigma_{p} / R^{2}\right) \delta\left(\lambda-\lambda_{0}\right) \sin \left(\phi-\phi_{0}\right)
$$

where $\lambda_{0}=72^{\circ} / 57.3^{\circ}, \alpha=6.75, \phi_{0}=0.45^{\circ}, \mathrm{R}=1 \mathrm{R}_{\mathrm{e}}, \Sigma_{\mathrm{p}}$ is the 
height-integrated Pedersen conductivity, and $\Phi_{0}$ is the same as in equation (9). Instead of postulating a driving field, equation (9), one could have obtained Figure 2 by postulating instead a driving current, equation (10); this was effectively the approach of IWASAKI and NISHIDA (1967), who obtained models similar to the present one.

Some magnetic observations recently reported by BEHANNON (1969) indicate that such field-aligned currents do exist near the outer boundary of the plasma sheet (which may reasonably be taken to be the boundary of "open" polar cap field lines). Behannon found a broad depressed field region within the magnetotail that appears to coincide with the plasma sheet; he also found that field lines in the magnetotail diverge away from the midnight meridian (interpreted as an expansion of the tail) and noted that this divergence is larger inside the depressed field region than outside. The latter observation implies that the current flowing on the boundary of the depressed field region has a field-aligned component, and working through the geometry one finds that this field-aligned current flows toward the earth in the dawn side of the tail and away from the earth in the dusk side, exactly in the directions required by Figure 2 and equation $(10)$.

The potential of Figure 2 was calculated assuming that the height-integrated conductivities are independent of latitude. Figure 3 shows the effect on the potential if the conductivity is enhanced at latitudes just below the polar cap, corresponding to the auroral 
zone. (Models with enhanced conductivity rings have been considered by IWASAKI and NISHIDA, 1967, WOLF, 1969, and, in a somewhat different context, by GOTTLIEB and FEJER, 1967, and SWIFT, 1968.) Specifically, both the height-integrated Hall and Pedersen conductivities were assumed to be increased by a factor of 10 between $\lambda=67^{\circ}$ and $\lambda=72^{\circ}$, maintaining the same ratio. The potential over the polar cap is unaffected (since equation (9) is still assumed to hold), while the potential pattern at latitudes below the enhanced conductivity ring is rotated eastward by $\sim 3^{\circ}$; the amount of this rotation (for which a simple physical explanation can be given) is primarily governed by the Hall to Pedersen conductivity ratio within the enhanced ring. The field-aligned current density is given by equation (10) with $\alpha=72.85, \phi_{0}=37.2^{\circ}$, and $\Sigma_{\mathrm{p}}$ the low latitude (not enhanced) value.

If the potential distribution of Figure 3 is mapped out to the equatorial plane, the transformation from a corotating to an inertial frame (i.e. the addition of the corotation electric field) will produce a region of closed flow lines near the earth which, following NISHIDA (1966) and BRICE (1967), is to be identified with the plasmasphere. The maximum radial extent of this region will here occur at a local time $\sim 37^{\circ}$ east of the dusk meridian, in qualitative agreement with the observed "bulge" of the plasmasphere (CARPENTER, 1966). (In the absence of an enhanced conductivity ring the maximum would occur on the dusk meridian itself; cf. Figure 2.) 
It is of interest to consider the ionospheric currents driven by the potential of Figure 3, and their magnetic effects. With the assumptions made, all current components are sinusoidal functions of longitude, with latitude-dependent amplitudes and phases. The amplitudes of the height-integrated east-west and north-south current density components and the phase of the east-west component are shown in Figure 4. The presence of the enhanced conductivity ring has produced two electrojets that bear a rather strong resemblance to the observed auroral electrojets. The uniform current over the polar cap is directed slightly east of the solar direction, as expected from the Hall current (along the equipotentials, away from the sun) and the Pedersen current (dawn to dusk); this is in disagreement with the "equivalent" current inferred from polar cap magnetic disturbances (e.g. SILSBEE and VESTINE, 1942; FAIRFIELD, 1963) which generally points well to the west of the sun.

However, it is meaningless to ask about the magnetic effects of a current system that is not closed, and hence the ionospheric current $\frac{I}{\sim}$ shown in Figure 4 cannot in any way be compared with "equivalent" currents constructed from observed magnetic variations. The current density $I$ can, nevertheless, be uniquely decomposed into a

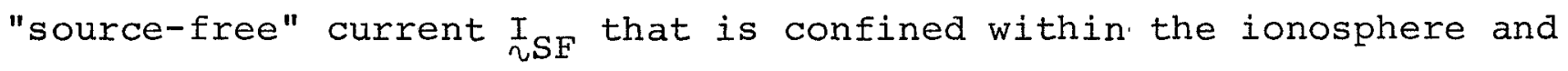
a current that serves merely to close the field-aligned currents: we write

$$
\underset{\sim}{I}=I_{\text {SF }}+\nabla \tau
$$


where by assumption $\nabla \cdot \tau_{\mathrm{SF}}=0$; the function $\tau$ can be calculated by inserting equation (11) into (7)

$$
\nabla^{2} \tau=J_{11} \sin x
$$

and $I_{\text {SF }}$ can then be obtained from (11). (In the absence of horizontal conductivity gradients, I $\mathrm{I} F$ is just the Hall current.) The ground level magnetic disturbance can then be considered as the sum of two contributions: (a) the magnetic field of the closed, purely ionospheric, current system $I_{\text {SFF }}$ (b) the magnetic field of the closed current system consisting of the ionospheric currents $\nabla \tau$, the field aligned currents and their associated magnetospheric currents, taken all together. (A third contribution, from closed currents flowing entirely within the magnetosphere, e.g. the symmetric part of the ring current, adds only a field essentially uniform over the earth.) If it is assumed that the ground-level magnetic field from current system (b) can be neglected, as suggested for instance by BOSTROM (1964) (and proved in the approximation of a plane ionosphere and straight field lines by FUKUSHIMA, 1968), then the current $I_{\text {SF }}$ is identical with the conventional "equivalent" current.

Figure 5 shows the source-free part ISSF of the ionospheric current driven by the potential of Figure 3. As can be seen, it bears a striking resemblance to the equivalent current of SILSBEE and VESTINE (1942), and in particular predicts the observed westward tilt 
over the polar cap (the tilt is a consequence of the enhanced conductivity ring, as can also be demonstrated by a simple qualitative argument). It should be clearly understood that in this model the equipotentials over the polar cap are still directed along the solar direction and the actual ionospheric current flows to the east of the sun, but a magnetometer on the polar cap observes a disturbance that, if attributed solely to an overhead sheet current, would imply a current direction tilted to the west; only the source-free part of the actual overhead current contributes to the magnetic field, the rest being assumed cancelled by effects of field-aligned and magnetospheric currents. These predictions of the model have been confirmed by the results of a polar cap barium cloud release reported at this conference by WESCOTT (1970), who observed that the plasma motion was approximately in the antisolar direction while the simultaneousIy observed ground-level magnetic disturbance corresponded to an overhead current directed $\sim 55^{\circ}$ west of the sun.

In summary, a very simple model of magnetospheric convection has been constructed, postulating a primary flow away from the sun over the polar cap and considering only the effects of the ionosphere. With the inclusion of an enhanced conductivity region at the auroral zones, this model predicts the observed location of the plasmasphere bulge and, with the assumption that "equivalent" currents constructed from magnetic disturbances primarily reflect the source-free component of the ionospheric current, it reproduces the main features of the 
DS current system, including in particular the westward tilt over the polar cap that had Iong been a problem for convection theories.

\section{Acknow ledgments}

I am grateful to $\mathrm{A}$. Nishida and $\mathrm{S}$. Olbert for valuable discussions. This research was supported by the National Aeronautics and Space Administration under grant NGR 22-009-015. 
REFERENCES

ALFVÉN, H.: 1939, Kungl. SV. Vet-Akademiens Handl. III, 18, 3.

AXFORD, W.I.: 1964, Plan. Space Sci. 12, 45.

AXFORD, W.I.: 1969 , Rev. Geophys. 7, 421.

AXFORD, W.I., and HINES, C.O.: 1961, Can. J. Phys. 39, 1433.

AXFORD, W.I., PETSCHEK, H.E., and SISCOE, G.L.: 1965, J. Geophys. Res. $70,1231$.

BEHANNON, K.W.: 1969, "Geometry of the Geomagnetic Tail", GSFC preprint $\mathrm{x}-616-69-146$.

BOSTRÖM, R.: 1964 , J. Geophys. Res. $\underbrace{69}, 4983$.

BRICE, N.M.: 1967, J. Geophys. Res. 72, 5193.

CARPENTER, D.L.: 1966, J. Geophys. Res. 71, 1675.

DUNGEY, J.W.: 1961, Phys. Rev. Lett. 6, 47. 
FAIRFIELD, D.H.: 1963, J. Geophys. Res. 68, 3589.

FEJER, J.A.: 1953, J. Atmosph. Terr. Phys. 4, 184.

FEJER, J.A.: 1964, J. Geophys. Res. 69, 123.

FUKUSHIMA, N.: 1968, "Equivalence in ground geomagnetic effect of Chapman - Vestine's and Birkeland - Alfvén's electric current systems for polar magnetic storms", preprint.

GOTTLIEB, B., and FEUER, J.A.: 1967, J. Geophys Res. 72, 239 .

IWASAKI, N., and NISHIDA, A.: 1967, Rept. Ionosph. Space Res. Japan 21,17 .

KAVANAGH, L.D., FREEMAN, J.W., and CHEN, A.J.: 1968, J. Geophys. Res. 73, 5511 .

KENNEL, C.F.: 1969, Rev. Geopinys. 7, 379.

KENNEL, C.F.: 1970, this volume.

NISHIDA, A.: 1966, J. Geophys. Res. 71, 5669. 
OBAYASHI, T., and NISHIDA, A.: 1968, Space Sci.Rev. $\underset{\sim}{ } 3$.

PARKER, E.N.: 1957, Phys. Rev. 107, 924.

PARKER, E.N.: 1968, Earth's Particles and Fields, (Ed. by B.M. McCormac), Reinhold Book Corporation, New York, 357.

SILSBEE, H.C., and VESTINE, E.H.: 1942, Terrest. Magnet. Atmosph. Elec. 47, 195 .

SISCOE, G.L.: 1964, "An Investigation of the Transport of Charged Particles in the Magnetosphere", Ph.D. Thesis, M.I.T. (unpublished).

SISCOE, G.L.: 1966, Plan. Space Sci. 14, 947.

SWIFT, D.W.: 1967, Plan. Space Sci. 15, 835.

SWIFT, D.W.: 1968, Plan. Space Sci. 16, 329.

TAYLOR, H.E., and HONES, E.W.: 1965, J. Geophys Res. 70, 3605.

VASYLIUNAS, V.M.: 1968, J.Geophys. Res. 73, 5805. 
VASYLIUNAS, V.M.; 1969, "A mathematical description of plasma motions in the magnetosphere", to be published.

WESCOTT, E.: 1970, this volume.

WOLF, R.A.: 1969, "Theoretical model for convective flow of plasma in the magnetosphere", preprint. 


\section{FIGURE CAPTIONS}

Figure 1. Outline of the self-consistent calculation of magnetospheric convection. The principal quantities being calculated are shown enclosed in boxes. Each line joining two boxes is labeled with the physical principle or equation that provides the link between the two quantities; the arrow points to that one of the two which can be calculated from the other. Quantities written in italics are boundary conditions that must be specified. ("Kinetic equation" is used as a general term to include whatever method for computing the particle distribution is deemed to be appropriate, e.g. Liouville's theorem, Boltzmann Vlasov equation and its transformations, etc.)

Figure 2. Calculated equipotential contours in the ionosphere, labeled by values in units of $\Phi_{0}$ (see text). Only the region above $50^{\circ}$ latitude is shown (the calculation, however was carried down to the equator and all magnetic field dip angle effects included). The outer circle is $\lambda=50^{\circ} ;$ the inner, $\lambda=72^{\circ}$.

Figure 3. Same as Figure 2 but including effects of enhanced conductivity between $\lambda=65^{\circ}$ and $72^{\circ}$. The three circles are $\lambda=50^{\circ}, 67^{\circ}$, and $72^{\circ}$, respectively. 
Figure 4. Actual height-integrated ionospheric current driven by the potential of Figure 3. Top: amplitude of the eastwest (EW) and north-south (NS) components of the current density, in units of $\Phi_{0} \Sigma_{p} / R$ (see text; $\Sigma_{p}$ is the low latitude value). Above $\lambda=72^{\circ}$ the $\mathrm{EW}$ and NS components have the same amplitude and differ in phase by $90^{\circ}$. Bottom: phase of the EW component; the dashed line indicates where the current changes from eastward to westward, and the arrows indicate the current direction. Solid circles (or portions thereof) are at $\lambda=50^{\circ}, 67^{\circ}$, and $72^{\circ}$.

Figure 5. Source-free part of the height-integrated ionospheric current driven by the potential of Figure 3 . Same description as for Figure 4. 


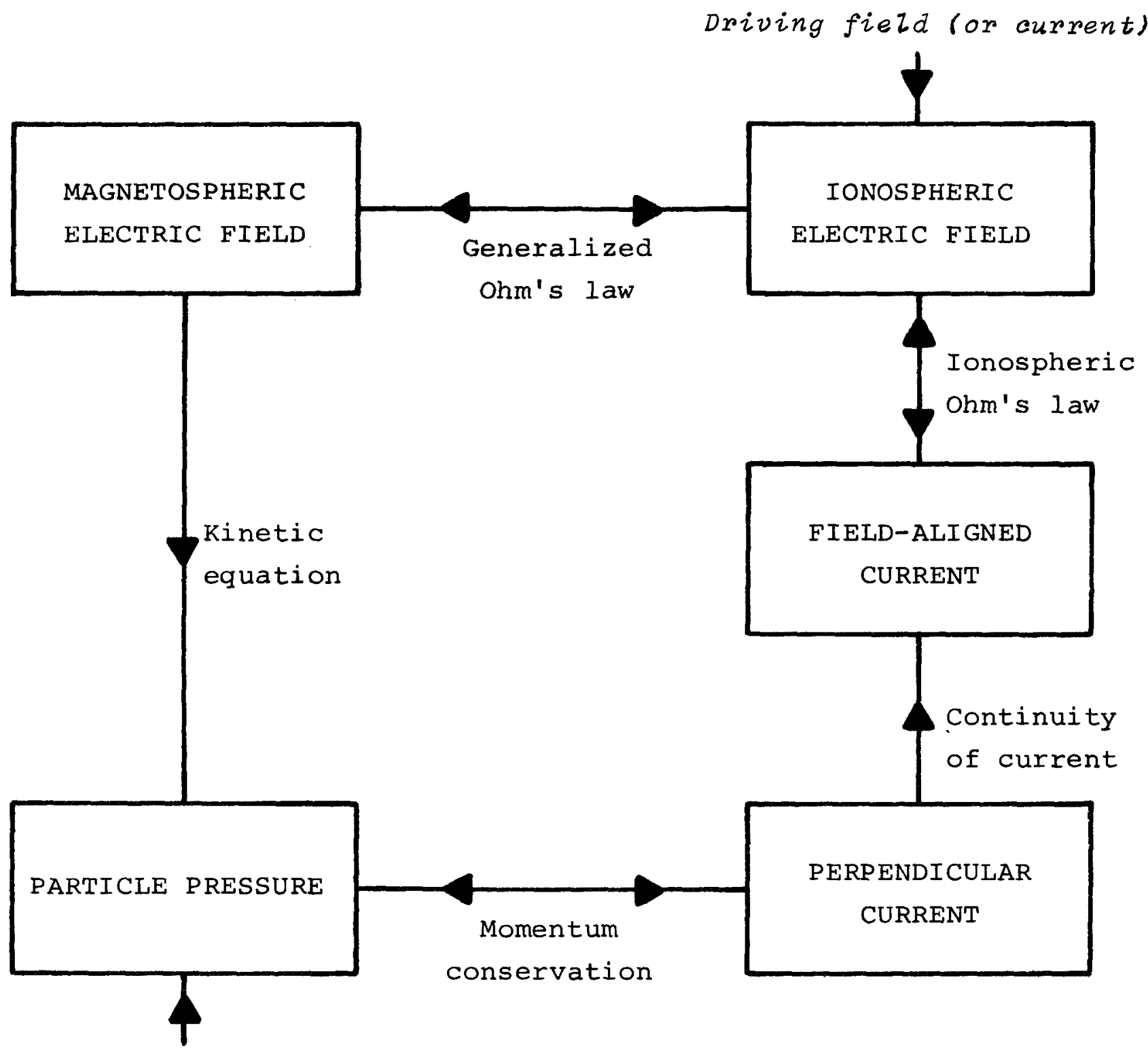

Boundary source

Figure 1 


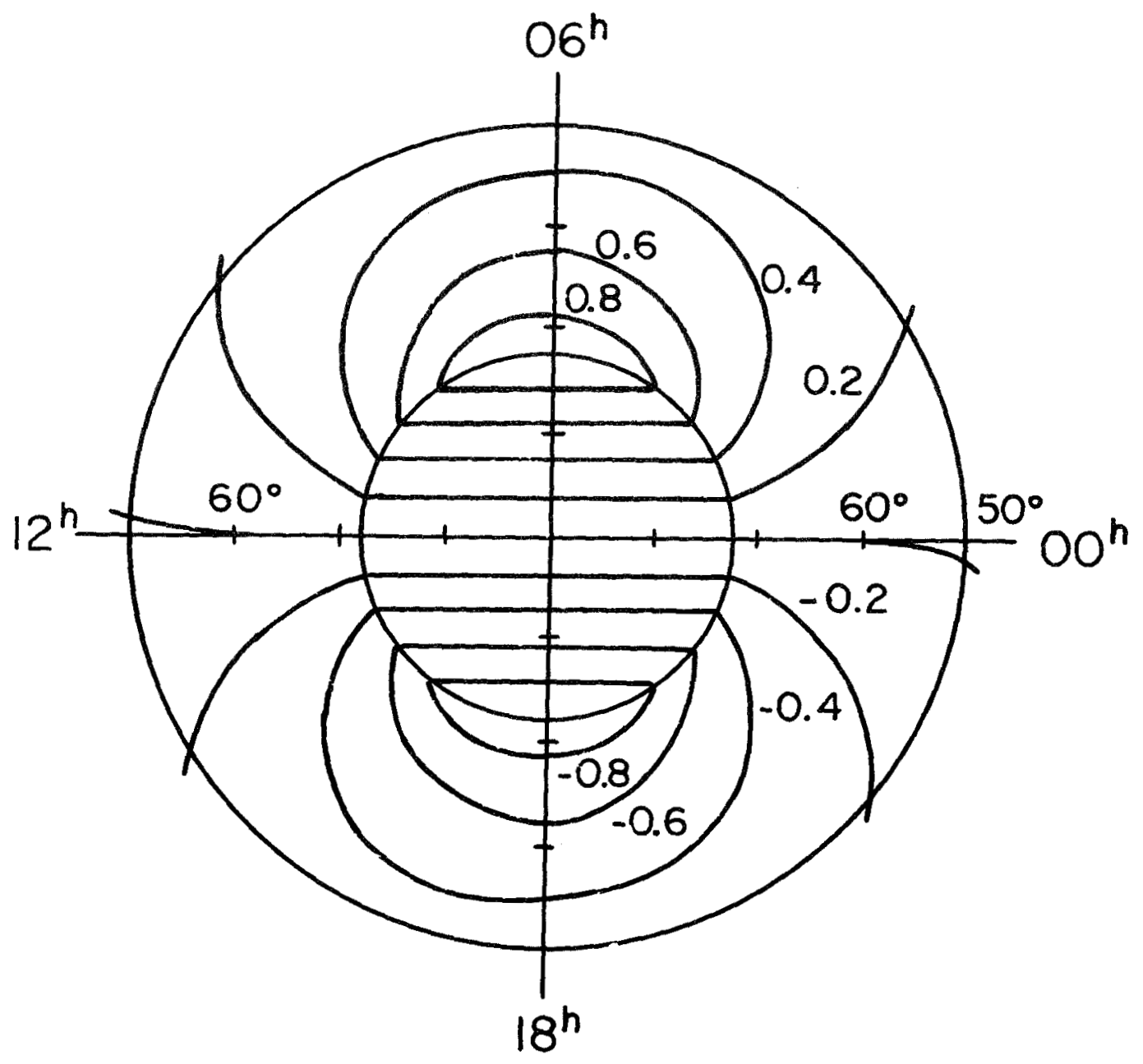

Figure 2 


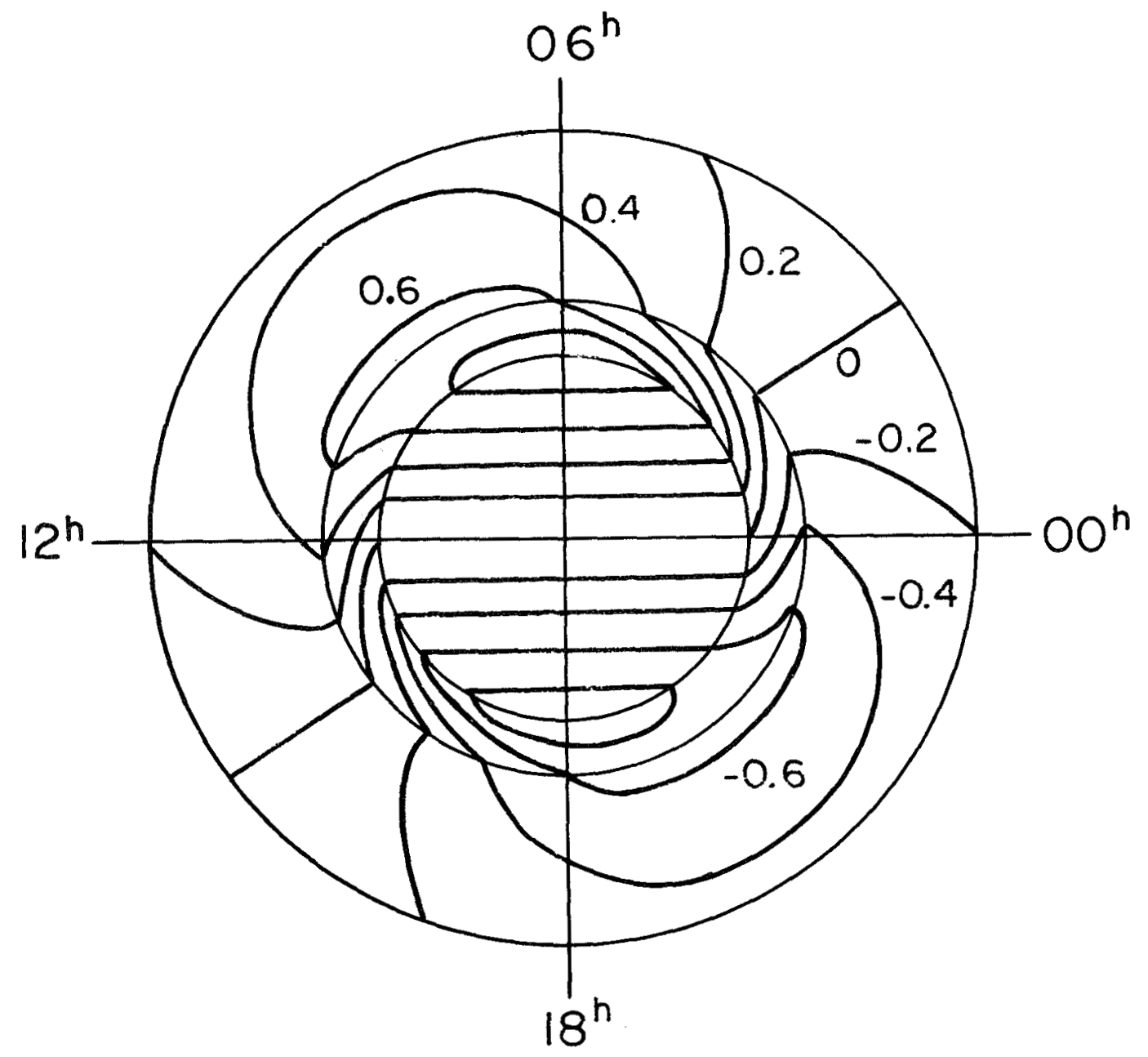

Figure 3 


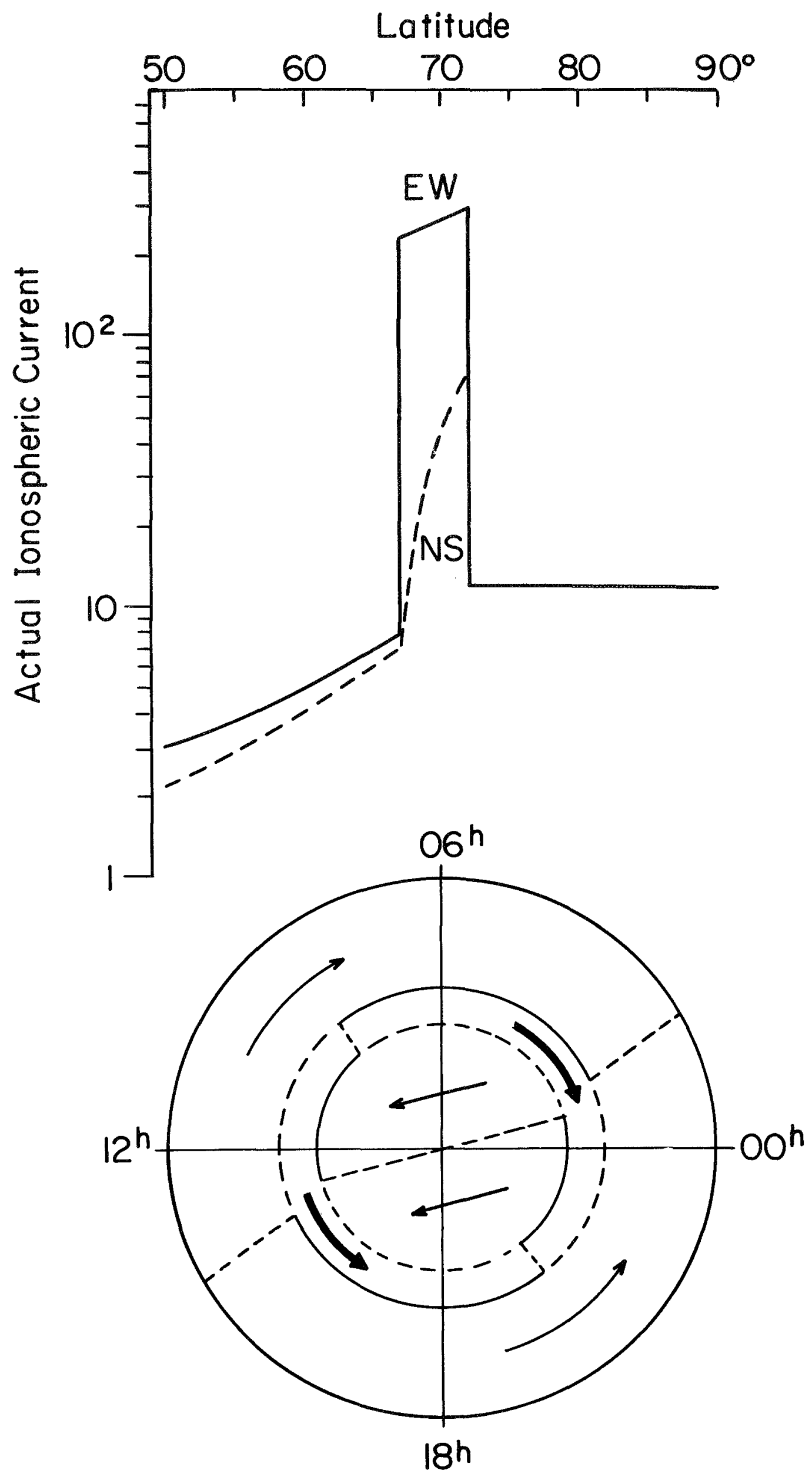

Figure 4 


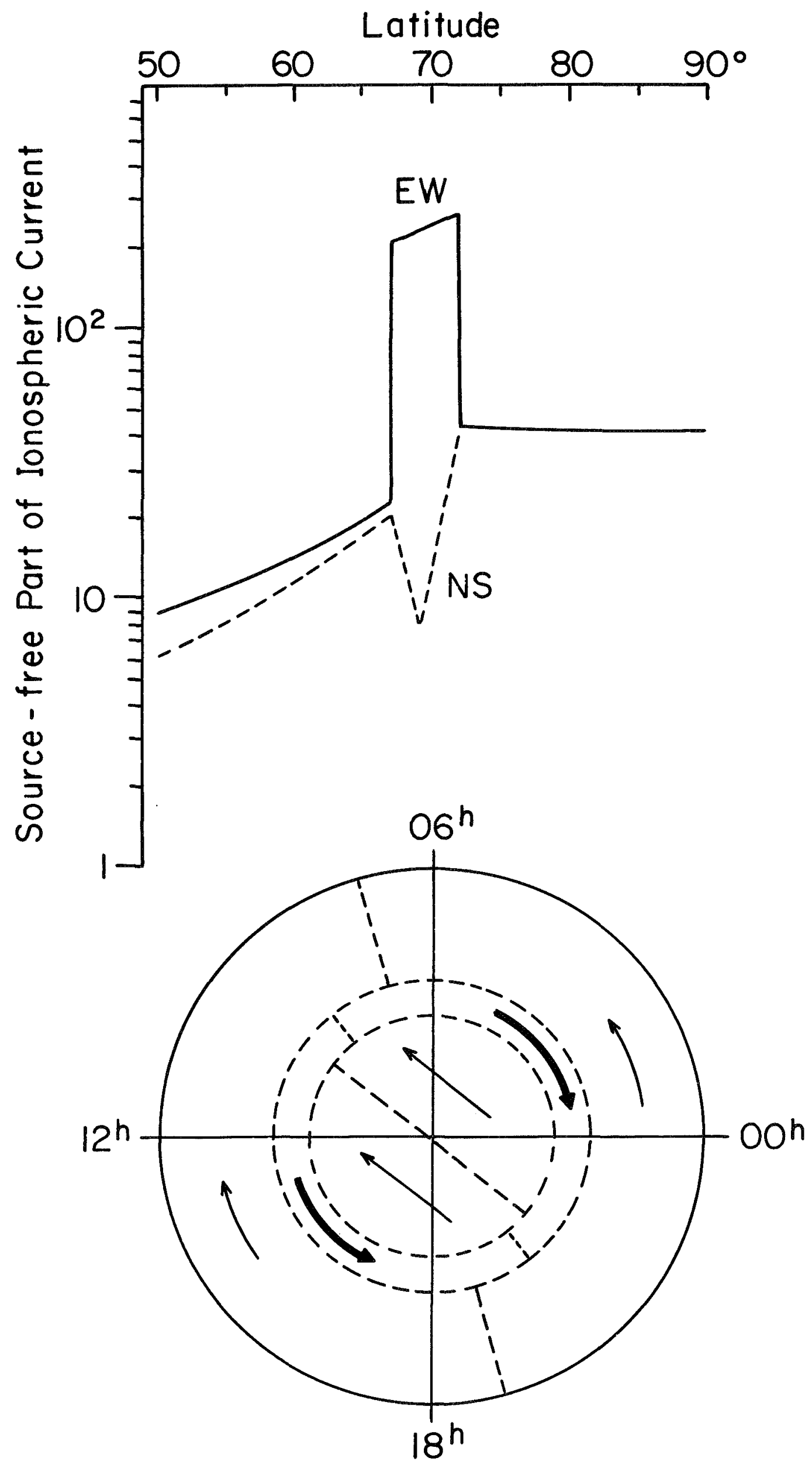

Figure 5 